University of Wollongong

Research Online

Faculty of Engineering and Information

Faculty of Engineering and Information

Sciences - Papers: Part B

Sciences

2018

\title{
On the first direct observation of de-twinning in a twinning-induced plasticity steel
}

Scott McCormack

University of Wollongong

Wei Wen

Prince of Wales Hospital

Elena V. Pereloma

University of Wollongong, elenap@uow.edu.au

Carlos N. Tome

Los Alamos National Laboratory

Azdiar Adil Gazder

University of Wollongong, azdiar@uow.edu.au

See next page for additional authors

Follow this and additional works at: https://ro.uow.edu.au/eispapers1

Part of the Engineering Commons, and the Science and Technology Studies Commons

Research Online is the open access institutional repository for the University of Wollongong. For further information contact the UOW Library: research-pubs@uow.edu.au 


\title{
On the first direct observation of de-twinning in a twinning-induced plasticity steel
}

\author{
Abstract \\ Electron back-scattering diffraction was used to track the microstructure evolution of a fully annealed \\ Fe-24Mn-3Al-2Si-1Ni-0.06C TWinning Induced Plasticity (TWIP) steel during interrupted reverse (tension- \\ compression) loading. Direct observation of the same selected area revealed that all deformation twins \\ formed during forward tension loading ( 0.128 true strain) were removed upon subsequent reverse \\ compression loading (0.031 true strain). Consequently, the present study provides the first unambiguous \\ experimental evidence of de-twinning during the reverse loading of a polycrystalline TWIP steel. The \\ reverse loading behaviour was simulated by a dislocation-based hardening model embedded in the Visco- \\ Plastic Self-Consistent (VPSC) polycrystal framework taking into account the accumulation and \\ annihilation of dislocations and back-stress effects. The model has been extended to account for the \\ processes of twinning and de-twinning, as well as the twin barrier effect under load reversal. A new \\ formulation based on the changes in the dislocation mean free path is proposed to track twin lamellae \\ generation/annihilation throughout deformation along with its associated effect on hardening.

\section{Disciplines} \\ Engineering | Science and Technology Studies \\ Publication Details \\ McCormack, S. J., Wen, W., Pereloma, E. V., Tome, C. N., Gazder, A. A. \& Saleh, A. A. (2018). On the first \\ direct observation of de-twinning in a twinning-induced plasticity steel. Acta Materialia, 156 172-182.

\section{Authors} \\ Scott McCormack, Wei Wen, Elena V. Pereloma, Carlos N. Tome, Azdiar Adil Gazder, and Ahmed A. Saleh
}




\title{
On the first direct observation of de-twinning in a twinning-induced plasticity steel
}

Scott J. McCormack¹, Wei Wen², Elena V. Pereloma1,3, Carlos N. Tomé2, Azdiar A. Gazder³, Ahmed A. Saleh ${ }^{1 *}$

${ }^{1}$ School of Mechanical, Materials and Mechatronic Engineering, University of Wollongong, New South Wales 2522, Australia

2Materials Science and Technology Division, Los Alamos National Laboratory, New Mexico 87545, United States

3Electron Microscopy Centre, University of Wollongong, New South Wales 2500, Australia

\begin{abstract}
Electron back-scattering diffraction was used to track the microstructure evolution of a fully annealed Fe24Mn-3Al-2Si-1Ni-0.06C TWinning Induced Plasticity (TWIP) steel during interrupted reverse (tensioncompression) loading. Direct observation of the same selected area revealed that all deformation twins formed during forward tension loading (0.128 true strain) were removed upon subsequent reverse compression loading ( 0.031 true strain). Consequently, the present study provides the first unambiguous experimental evidence of de-twinning during the reverse loading of a polycrystalline TWIP steel. The reverse loading behaviour was simulated by a dislocation-based hardening model embedded in the ViscoPlastic Self-Consistent (VPSC) polycrystal framework taking into account the accumulation and annihilation of dislocations and back-stress effects. The model has been extended to account for the processes of twinning and de-twinning, as well as the twin barrier effect under load reversal. A new formulation based on the changes in the dislocation mean free path is proposed to track twin lamellae generation/annihilation throughout deformation along with its associated effect on hardening.
\end{abstract}

Keywords: TWIP steel, de-twinning, reverse loading, electron back-scattering diffraction (EBSD), VPSC.

*Corresponding author:

Ahmed A. Saleh

School of Mechanical, Materials and Mechatronic Engineering

University of Wollongong, New South Wales 2522, Australia

Phone: +61242213034 Fax: +61242213114ｅ-mail: asaleh@uow.edu.au 


\section{Introduction}

Twinning-induced plasticity (TWIP) steels containing 25-35 wt.\% Mn with small additions of $\mathrm{Al}$ and Si are stable face-centred cubic (fcc) austenitic steels with low stacking fault energy (SFE = 15-40 $\mathrm{mJ} \mathrm{m}^{-2}$ ) [1]. Upon the imposition of external strain, the low SFE promotes deformation accommodation via twinning along with a combination of perfect and partial slip. Here the dissociation of perfect dislocations gliding on the $\{111\}$ plane in the $\langle 110\rangle$ direction results in two $\langle 112\rangle$ Shockley partials bounding stacking faults [2]. The equilibrium separation distance between the two partials is inversely proportional to the SFE of the material. However this separation distance may increase or decrease (as the partials are pulled apart or pushed together) during deformation, depending on the direction of the applied load and the grain orientation [3-5]. A deformation twin forms when the dissociated partials diverge from each other to an infinite separation distance [2].

It is common knowledge that in hexagonal close packed (hcp) materials [6-10], deformation twins undergo de-twinning when loaded in the reverse direction at room temperature, thereby causing partial or complete disappearance (or de-twinning) of the twinned region. For example, de-twinning of tensile twins was reported in $\mathrm{Mg}$ [6] and $\mathrm{Zr}$ [7] such that twins alternately disappear (or become narrower) and reappear during subsequent unloading and reloading cycles, respectively. This effect is ascribed to the back stress that the shear transformation induces in the vicinity of the twin domain [11-16]. For instance, in the case of tension-compression reverse loading, the twinning-induced back stress during tension adds to the resolved shear stress component of the applied compression stress upon load reversal, which in turn facilitates the de-twinning process. .

In contrast to the large body of experimental work on de-twinning in hcp materials, very few studies have been undertaken on cubic materials. For example, de-twinning was observed during the cyclic loading of body-centred cubic Ti-V alloys [17] and in nanocrystalline fcc alloys subjected to monotonic loading to high strains. In the latter case, for electro-deposited nanocrystalline $\mathrm{Ni}$-20Fe with pre-existing growth twins subjected to room temperature $6 \mathrm{GPa}$ high-pressure torsion, it was found that for 10-45 $\mathrm{nm}$ grain sizes, detwinning of existing growth and deformation twins prevailed over the formation of new deformation twins [18]. Similarly, nanocrystalline Ni-18Fe fabricated by pulsed electro-chemical deposition subjected to $82 \%$ and $77 \%$ rolling reductions at room and liquid nitrogen temperatures, respectively, exhibited a reduction in twin densities [19]. However, the deformation mechanisms that led to de-twinning in nanocrystalline fcc alloys are significantly different compared to their coarse (micron-sized) counterparts.

In $\mathrm{Cu}-8.5 \mathrm{Al}$ fcc single crystals [20], de-twinning was observed when compression was applied after the generation of deformation twins during pre-loading in tension. In one of the only studies pointing out the possibility of de-twinning in coarse grained polycrystalline fcc materials, Doquet [21] detected fragmented twins after reverse torsion of low SFE Co-33Ni alloy. Following Ref. [17], Doquet argued that the observed 
twin fragmentation upon load reversal was due to the interaction between dislocations and twins along the twin-matrix interface which does not allow for the homogenous de-twinning of the entire twin.

Overall, since twinning introduces a large localized shear in the grain, it is always accompanied by the reaction of the surrounding medium, in the form of a back-shear stress. This back-shear stress has been documented computationally and experimentally in Refs. [12, 22, 23] and tends to favour de-twinning upon unloading or load reversal, independently of the specific crystallography of the system. In this regard, while several models (such as the visco-plastic [24] and elasto-visco-plastic [8, 10, 25] self-consistent models and the Taylor-Lin elasto-visco-plastic model [26]) have been recently developed and adapted to simulate the de-twinning process in hcp materials, no modelling effort has been directed towards fcc (or cubic) materials to date.

To this end, in a recent elasto-plastic self-consistent (EPSC) modelling study, Saleh et al. [27] highlighted the feasibility of de-twinning during the reverse loading of a Fe-24Mn-3Al-2Si-1 Ni-0.06C TWIP steel subjected to $\pm 1 \%$ cyclic (tension-compression) loading. However, to the best of our knowledge, there is no unambiguous experimental observation of de-twinning in coarse-grained (micron-sized) polycrystalline fcc alloys to-date

The present study provides first-time evidence of de-twinning during the reverse loading of a polycrystalline TWIP steel using electron back-scattering diffraction (EBSD). It is also the first study that interprets the experimental evidence in terms of a dislocation-based hardening model embedded in the visco-plastic self-consistent (VPSC) framework that accounts for the accumulation and annihilation of dislocations and back-stress effects. The model has been modified (as detailed in Section 3) in order to explicitly captures the twinning and de-twinning processes during load reversal.

\section{Experimental procedure}

A slab-cast TWIP steel (Fe-24Mn-3Al-2Si-0.9Ni-0.05C wt.\%) was 52\% hot rolled and 42\% cold rolled. A dog-bone shaped tension-compression sample with 4 (gauge length) $\times 4$ (width) $\times 4$ (thickness) $\mathrm{mm}^{3}$ was wire-cut from the middle of the cold-rolled strip keeping its gauge length parallel to the rolling direction (Fig. 1). The dimensions of the gauge length, width and thickness of the dog-bone sample were deliberately selected to avoid buckling during tension-compression loading. The dog-bone sample was annealed at 850 ${ }^{\circ} \mathrm{C}$, which included $240 \mathrm{~s}$ of heating to a stable temperature followed by $300 \mathrm{~s}$ of soaking time and immediate water quenching. 
Uniaxial tension-compression was undertaken on a servo-hydraulic universal tester operating in speed control mode at $0.005 \mathrm{~mm} / \mathrm{s}$. The test was interrupted at true strains ${ }^{1}$ of 0.128 in tension followed by reverse loading in compression back to a true strain of 0.031 .

EBSD was undertaken on a JEOL-JSM7001F field emission gun-scanning electron microscope fitted with a Nordlys-II (S) detector and interfacing with the Oxford Instruments AZtec acquisition software; operating at $15 \mathrm{kV}$ accelerating voltage, $3 \mathrm{nA}$ probe current and $15 \mathrm{~mm}$ working distance. EBSD maps of a selected area located in the middle of the gauge length were collected at true strains of 0 (initial), 0.128 (after forward tension loading, T) and 0.031 (after reverse compression loading, TC), using a constant step size of $0.1 \mu \mathrm{m}$.

In all maps, a minimum of 3 pixels was used to identify grain structures. Low-angle grain boundaries (LAGBs, shown in blue) are defined as $2^{\circ} \leq \theta<15^{\circ}$ misorientations, while high-angle grain boundaries (HAGBs, shown in black) comprise misorientations with $\theta \geq 15^{\circ}$. First and second order twin boundaries (TBs) are defined as $\Sigma 3=60^{\circ}\langle 111\rangle$ (shown in red) and $\Sigma 9=38.9^{\circ}\langle 110\rangle$ (shown in green). The maximum tolerance of the misorientation angle $(\Delta \theta)$ from the exact axis-angle relationship was indentified following the Palumbo-Aust criterion (i.e.- $\Delta \theta \leq 15^{\circ} \Sigma^{-5 / 6}$ ] [28], yielding tolerance limits of $6^{\circ}$ for $\Sigma 3$ and $2.4^{\circ}$ for $\Sigma 9$.

\section{Visco-plastic self-consistent modelling}

In 2011, Rauch-Gracio-Barlat-Vincze (RGBV) [29] proposed a continuum plasticity model to account for strain path change effects on the stress-strain response of solids. This model is based on the concept that some of the dislocations generated during loading in one direction, namely reversible dislocations, can reverse their glide direction and progressively recombine upon load reversal. In 2013, Kitayama et al. [30] explicitly accounted for the change in strain path by reformulating the RGBV model for a crystallographic framework, which allows identification of the shear reversal on each slip system within every grain. The crystallographic RGBV model was extended by Wen et al. to predict the mechanical response associated with tension reloading of AZ31 Mg alloy [31] and cyclic shear tests of low-carbon steel [32]. However, these applications do not involve deformation twinning. The VPSC model used in this study is based on the RGBVVPSC framework [30-32] and modified to account for the processes of twinning and de-twinning, as well as the twin barrier effect under strain path change. While the inclusion formalism of the VPSC model inherently captures the effect of intergranular stresses, the empirical back stress equations introduced in the present work for both slip and twinning account for the intragranular stresses. Twin lamellae generation/annihilation is tracked throughout deformation and its effect on hardening is formulated via the changes in dislocation mean free path. The RGBV-VPSC formulation is detailed in Ref. [32] and only a brief

${ }^{1}$ All stresses and strains are reported as true stresses and strains, unless otherwise stated. 
description of the hardening at the single crystal level is given is Section 3.1. Thereafter Sections 3.2 and 3.3 detail the new twinning/de-twinning formulation and the twin barrier effect, respectively.

\subsection{Introduction to the RGBV model}

In TWIP steel, deformation twins present as twin lamellae that bundle together to act as strong obstacles to dislocation glide via a twin barrier effect. In this work, the hardening of slip due to the sliptwinning interaction is modelled assuming the twin lamellae may: (i) reduce the dislocation mean free path, which alters the dislocation accumulation rate, and (ii) harden the slip systems directly via the Hall-Petch effect. Thus, the critical resolved shear stress (CRSS) in a slip system $s$ is written as:

$$
\tau_{c}^{S}=\tau_{0}^{S}+\tau_{d}^{S}+\Delta \tau_{B}^{S}+\tau_{H P}^{S}
$$

Here, $\tau_{0}^{S}$ represents the hardening contributions from lattice friction and pre-existing dislocations. Following Beyerlein and Tomé [33], $\tau_{H P}^{S}$ is the Hall-Petch term which depends on the dislocation mean free path for a slip system $s$ :

$$
\tau_{H P}^{S}=\mu H P^{s} \sqrt{\frac{b^{s}}{d_{m f p}^{S}}}
$$

where, $\mu$ is the shear modulus and $b^{s}$ is the magnitude of Burger's vector. $H P^{s}$ refers to the Hall-Petch coefficient describing the slip/barriers interaction strength. $d_{m f p}^{s}$ is the dislocation mean free path for a slip system $s$ and is a function of the spacing between hard barriers. Here barriers refer to the generated deformation twins and pre-existing barriers such as grain boundaries and annealing twins. Since all such features are strong barriers to dislocation glide [34], their interaction strength $\left(H P^{s}\right)$ with slip is assumed to be equal. The equation for $d_{m f p}^{s}$ is detailed in Section 3.3.

$\tau_{d}^{s}$ in Eq. (1) describes the hardening contribution of dislocation accumulation/annihilation. It is calculated through the coupled form of Taylor law [35-38] written as:

$$
\tau_{d}^{s}=\mu b^{s} \sqrt{\sum_{s} \alpha^{s s^{\prime}} \rho^{s^{\prime}}}
$$

$\alpha^{s s^{\prime}}$ is the effective latent hardening matrix while $\rho^{s}$ refers to the total dislocation density in a slip system $s$. In the crystallographic RGBV model, the dislocations in each system are split into forest (nonreversible) and reversible dislocations. The latter dislocations recombine when the shear is reversed. In order to account for directionality, each slip system is divided into $s^{+}$and $s^{-}$, which correspond to the activation of a slip system in positive and negative directions (defined arbitrarily). Therefore, $\rho^{s}$ is given as:

$$
\rho^{s}=\rho_{\text {for }}^{s}+\rho_{\text {rev }}^{s+}+\rho_{\text {rev }}^{s-}
$$


where, $\rho_{\text {for }}^{S}$ is the forest dislocation density. $\rho_{\text {rev }}^{S+}$ and $\rho_{\text {rev }}^{S-}$ denote the reversible dislocation densities on $s^{+}$and $s^{-}$, respectively. The evolution laws for $\rho_{\text {for }}^{s}, \rho_{\text {rev }}^{s+}$ and $\rho_{\text {rev }}^{s-}$ rely on the Kocks-Mecking formulation [39]. If the slip system shears in the positive direction $\left(d \gamma^{s+}>0 ; d \gamma^{s-}=0\right), \rho_{\text {for }}^{s}$ and $\rho_{\text {rev }}^{s+}$ evolve via storage and thermally-activated recovery terms:

$$
\begin{gathered}
d \rho_{\text {for }}^{s}=\left(1-P^{s}\right) \frac{d \gamma^{s+}}{b \Lambda}-f \rho_{\text {for }}^{s} d \Gamma \\
d \rho_{\text {rev }}^{s+}=P^{s} \frac{d \gamma^{s+}}{b \Lambda}-f \rho_{\text {rev }}^{s+} d \Gamma
\end{gathered}
$$

where, $f$ denotes the recovery coefficient. In general, $f$ is a function of rate and temperature, but here it is treated as a constant, since there is no variation of rate or temperature. $d \Gamma=\sum_{s}\left|d \gamma^{s}\right|$ is used in the recovery term instead of $d \gamma^{s+}$ since the activation of any slip system will, in general, facilitate dislocation annihilation in system $s$. In Eq. (5), $\Lambda$ denotes the effective dislocation mean free path and is different from $d_{m f p}^{s}$ (Eq. (2)) as it denotes both, the spacing between weak dislocation obstacles and strong obstacles. Therefore, $\Lambda$ can be determined from the harmonic mean as follows:

$$
\frac{1}{\Lambda^{s}}=\frac{\sqrt{\rho}}{K}+\frac{1}{d_{m f p}^{S}}
$$

where, $K$ is the number of dislocation-type obstacles that a moving segment is able to cross before being trapped. $\rho$ is the total dislocation density in one grain. $P^{s}$ in Eq. (5) denotes the reversibility parameter controlling the fraction of generated dislocations that are reversible.

In the original version of the RGBV model [30], this parameter decreases monotonically from 1 to 0 with increasing accumulated strain. Recently, Wen et al. [32] modified the formulation, by allowing for the calculation of $P^{S}$ individually on each slip system, in order to account for dislocation recovery in individual systems. Doing so helps to saturate the mechanical response under cyclic loading to large accumulated strains. The evolution law for $P^{s}$ is described empirically by the sigmoidal function:

$$
P^{s}\left(D^{s}\right)=\frac{1}{2}-\frac{1}{2} \tanh \left[3\left(\frac{\rho_{\max }+\rho_{\min }}{\rho_{\max }-\rho_{\min }}\right)\left(\frac{\sqrt{D^{s}}-\rho_{\text {infl }}}{\rho_{\text {infl }}}\right)\right]
$$

where, $\rho_{\max }$ and $\rho_{\min }$ are characteristic densities and $\rho_{\text {infl }}=\left(\rho_{\max }+\rho_{\min }\right) / 2$ is the inflection point of the sigmoidal function. $\sqrt{D^{s}}$ is a measure of the dislocation density blocking dislocation reversal on a slip system $s$ and is assumed to evolve incrementally as follows:

$$
D^{s}=\int_{0}^{\varepsilon} \Delta D^{s}
$$

where, 


$$
\Delta D^{s}=\Delta \rho^{s} \sum_{s^{\prime}} \rho^{s^{\prime}}\left(1-\left|\bar{n}^{s} \cdot \bar{n}^{s^{\prime}}\right|\right)
$$

The factor $\left(1-\left|\bar{n}^{s} \cdot \bar{n}^{s^{\prime}}\right|\right)=1-\cos \left(\bar{n}^{s} \cdot \bar{n}^{s^{\prime}}\right)$ describes the slip-slip interaction strength, which controls the coupling between $s$ and $s^{\prime}$ in a simple manner without detailing dislocation junctions. While $\rho_{\text {for }}^{S}$ and $\rho_{\text {rev }}^{S+}$ accumulate through the Kocks-Mecking law (Eq.5), the previously generated $\rho_{\text {rev }}^{S-}$ (if present non-zero) will be gradually recombined following:

$$
d \rho_{\text {rev }}^{s-}=-\frac{1}{b \Lambda}\left(\frac{\rho_{\text {rev }}^{s-}}{\rho_{0}^{s}}\right)^{m} d \gamma^{s+}
$$

In Eq. (10), $\rho_{0}^{S}$ is the total dislocation density in the system at the point of shear reversal. $0<m<1$ was originally introduced by Wen et al. [31] to achieve a faster recombination rate. In the RGBV model, the activation of slip in both directions is equivalent. Therefore, the activation of a slip system $s$ in the negative direction $\left(d \gamma^{s-}>0 ; d \gamma^{s+}=0\right)$ results in:

$$
\begin{gathered}
d \rho_{\text {for }}^{s}=\left(1-P^{s}\right) \frac{d \gamma^{s-}}{b \Lambda}-f \rho_{\text {for }}^{s} d \Gamma \\
d \rho_{\text {rev }}^{s+}=-\frac{1}{b \Lambda}\left(\frac{\rho_{\text {rev }}^{s+}}{\rho_{0}^{s}}\right)^{m} d \gamma^{s-} \\
d \rho_{\text {rev }}^{s-}=P^{s} \frac{d \gamma^{s-}}{b \Lambda}-f \rho_{\text {rev }}^{s-} d \Gamma
\end{gathered}
$$

Besides the dislocation accumulation/annihilation and the Hall-Petch effect, the hardening evolution of slip systems is also associated with a back-stress ( $\Delta \tau_{B}^{S}$ in Eq. (1)). In the RGBV model, the back-stress law is based on the long-range intragranular stress theory. During forward loading, polarised internal stresses are generated due to the formation of dislocation pile-ups. Upon load reversal, the back- stress assists in the re-mobilisation of reversible dislocations in the opposite direction with a lower resistance, which in turn, leads to a drop in the macroscopic yield stress (commonly referred to as the Bauschinger effect). Upon continued loading in the reverse direction, the dislocations gliding in the reverse direction are progressively recombined, which favours the relaxation of the residual stresses inherited from forward loading. Consequently, the back-stress effect reduces gradually and can potentially vanish. The expression for $\Delta \tau_{B}^{S}$ in the RGBV model is an empirical power law:

$$
\begin{array}{ll}
\Delta \tau_{B}^{S}=-\tau_{d}^{s} f_{B}^{s}\left(\frac{\rho_{\text {rev }}^{s-}}{\rho^{s}}\right)^{q} & \text { if } d \gamma^{s+}>0 \\
\Delta \tau_{B}^{S}=-\tau_{d}^{s} f_{B}^{s}\left(\frac{\rho_{\text {rev }}^{s+}}{\rho^{s}}\right)^{q} & \text { if } d \gamma^{s-}>0
\end{array}
$$

Here the residual back-stress is linked to the reversible dislocation density. According to Eqs. (5) and (10), $\rho_{\text {rev }}^{s+}$ or $\rho_{\text {rev }}^{s-}$ tends to recombine during shear reversal. It follows that the contribution of back-stress decreases gradually and eventually vanishes at a certain amount of reverse strain. 


\subsection{Hardening of twinning systems}

In the classic VPSC modelling framework $[40,41]$, twin systems are treated similar to slip systems in that their shear rates are also determined by the visco-plastic power law, with an added directionality condition that prevents the activation of twin systems in the negative direction. In this work, the treatment remains, with modifications that allow for negative shear during the de-twinning process (refer to Section 3.3 for details).

The hardening of twin systems is still described by the evolution of their threshold stress for activation. Here we employ the law proposed by Beyerlein and Tomé [33] with an extra back-stress term to account for the effect of interface separation caused by the formation of twin lamellae:

$$
\tau^{t}=\tau_{0}^{t}+\tau_{d}^{t}+\tau_{H P}^{t}+\Delta \tau_{B}^{t}
$$

Here, $\tau_{0}^{t}$ is the initial CRSS of a twin system $t$ without any contributions from the surrounding dislocation structure $\left(\tau_{d}^{t}\right)$, the Hall-Petch effect $\left(\tau_{H P}^{t}\right)$ or the back-stress $\left(\Delta \tau_{B}^{t}\right)$. In Ref. [33], $\tau_{d}^{t}$ is defined as:

$$
\tau_{d}^{t}=\mu \sum_{s} C^{t s} b^{t} b^{s} \rho^{s}
$$

Here, $C^{t s}$ is related to the interaction strength between a slip system $s$ and a twin system $t$. The HallPetch term $\left(\tau_{H P}^{t}\right)$ is given as:

$$
\begin{aligned}
& \tau_{H P}^{t}=\frac{H P^{t}}{\sqrt{D}} \text { if only parallel twin lamellae are present } \\
& \tau_{H P}^{t}=\frac{H P^{t t^{\prime}}}{\sqrt{d_{m f p}^{S}}} \text { if otherwise }
\end{aligned}
$$

In contrast to dislocation slip, deformation twins accommodate the strain by creating lamellar domains associated with lattice reorientation and localised twinning shear. For example, twinning in fcc materials results in a characteristic twinning shear $S=0.707$. The surrounding matrix constrains the localised twining shear and introduces stress reversal in the twinned region [22]. The amount of shear stress reversal depends on elastic and plastic anisotropy and the orientations of neighbouring grains, as detailed in hcp materials [11-14]. The stress reversal, defined as the difference between the resolved shear stresses on the twin plane before and after twin formation, suppresses twin thickening upon further loading and favours de-twinning upon load reversal. In the VPSC framework, the twinning shear is explicitly accounted for, but not the twinning back-stress induced by neighbouring grains. Recently, Arul Kumar et al. [42] used a fullfield Fast Fourier Transform model to calculate the twinning back-stress as a function of neighbouring grain orientations and implemented it statistically in the VPSC framework for tensile twins in hcp materials. The results evidenced the influence of twinning back-stress correction on twin thickening in the VPSC framework. 
In contrast to hcp materials, while twin thickening is not an important consideration in the present low SFE fcc TWIP steel, de-twinning during load reversal is of relevance. Consequently, and similar to Clausen et al. [43], we account for the twinning back-stress in an ad-hoc manner without directly including neighbouring grain dependence.

A recent body of experimental and numerical work in hcp materials demonstrates that the twin shear transformation induces important back-stresses inside the twin domain, in the vicinity of the twin in the parent grain, and in the neighbouring grains when the twin terminates at grain boundaries [11-16]. Ref. [36] shows that increasing the number of twin lamellae and/or decreasing their separation tends to increase the magnitude of the overall back-stress. We expect a qualitatively similar situation to take place in cubic materials with twins. Based on these observations, we propose a simple empirical estimate for the twinning back-stress associated with twins in our TWIP steel, as follows:

$$
\Delta \tau_{B}^{t}=\varphi \mu S \frac{N^{t} d_{t}}{D}
$$

where the shear modulus $\mu=76 \mathrm{GPa}$ and the characteristic twinning shear $S=0.707$ as mentioned above. The role of Eq. 16 is to 'spread' $S$ proportionally to the twinned fraction of the grain. $\varphi$ is a fitting parameter that quantifies the fraction of the total shear transformation that is accommodated elastically and will induce back-stress. In our case, the fitting returns a value of $\varphi=0.0186$. The twinning back-stress has to be regarded as an effective magnitude evolving with the twin area fraction $\left(d_{t} / D\right)$ and the number of twins $\left(N^{t}\right)$.

\subsection{Twinning/de-twinning and the twin barrier effect}

As opposed to twin propagation in hcp materials, TWIP steels tend to form new fine twin lamellae with thicknesses typically ranging from 10 to $30 \mathrm{~nm}$ during loading $[34,44]$. The newly generated twin lamellae act as obstacles to plastic flow and enhance the hardening behaviour of the material. In the present model,

this twin barrier effect is accounted for through $d_{m f p}^{S}$, which is controlled by two factors: (i) the interspacing between neighbouring twins, and (ii) their geometrical relationship with the slip plane. While individual twin interspacing tends to be heterogeneous, it is sufficient to use an effective average twin interspacing during constitutive modelling. If we assume that the number of twins contained in one grain is $N^{t}$, the parent matrix is equally divided into $N^{t}+1$ parts as shown schematically in Fig. 2a. For the activated twinning systems, $N^{t}$ is allowed to gradually increase during loading and decrease upon shear reversal; such that it mimics the processes of twinning and de-twinning, where twins appear or disappear in succession and not simultaneously. The determination of $N^{t}$ relies on the associated twin volume fraction: 


$$
f^{t}=\frac{\gamma^{t}}{S}
$$

where, $\gamma^{t}$ is the accumulated shear on a twinning system $t$. In applications of the VPSC model [40, 41], the activation of twinning in the negative direction is prohibited by enforcing the condition that the twin shear rate $\dot{\gamma}^{t} \geq 0$. Accordingly, in the present work, $\dot{\gamma}^{t} \geq 0$ is enforced for the activation and growth of twinning in the parent grain. However, since de-twinning corresponds to $\dot{\gamma}^{t} \geq 0$ in the twinned volume and $\dot{\gamma}^{t}<0$ in the parent, in the current application we calculate the resolved shear on the twin plane using the stress of the parent, and activate de-twinning when $\dot{\gamma}^{t}<0$ in the parent. We stop applying such procedure once/if all twins have de-twinned and recovered the parent orientation.

The number of twin lamellae $N^{t}$ is associated with $f^{t}\left(\gamma^{t}\right)$ and is initially zero. It is assumed that one twin lamellae will be created when $f^{t}$ reaches the volume fraction of one twin lamellae $\left(d_{t} / D\right)$, where $d_{t}$ is the average twin lamellae thickness. In this manner, $N^{t}$ is allowed to increase during forward loading and progressively decrease upon shear reversal until it reaches zero. The expression for $N^{t}$ is written as:

$$
N^{t}=\operatorname{int}\left[\frac{f^{t}}{\left(d_{t} / D\right)}\right]
$$

Here, the int[] function rounds a number to the lower integer. Once $N^{t}$ is calculated, the twin interspacing $\lambda^{t}$ (see Fig. 2a) is determined as:

$$
\lambda^{t}=\frac{\left(D-N^{t} d_{t}\right)}{\left(N^{t}+1\right)}
$$

The evolution of the average number of twin lamellae per grain and their average interspacing as a function of the associated twin volume fraction is illustrated in Fig. $2 b$ during forward loading of our aggregate. The geometrical relationship giving the mean free path for the activated twin systems (Fig. 2a) is given by:

$$
d_{m f p}^{s, t}=\frac{\lambda^{t}}{\sin \alpha}
$$

where, $\alpha=\arccos \left(\left|\overline{\boldsymbol{n}}^{s} \cdot \overline{\boldsymbol{n}}^{t}\right|\right)$ is the angle between the slip and twinning planes. $\overline{\boldsymbol{n}}^{s}$ and $\overline{\boldsymbol{n}}^{t}$ are the normal vectors for the slip and twinning planes, respectively. Note that the activation of more than one twinning system is allowed here. In this case, the effective mean free path $d_{m f p}^{s}$ for a slip system $s$ is determined as the harmonic mean of $d_{m f p}^{s, t}$ for all systems that formed twin lamellae:

$$
\frac{1}{d_{m f p}^{s}}=\frac{1}{d_{m f p}^{s, t_{1}}}+\frac{1}{d_{m f p}^{s, t_{2}}}+\cdots
$$

Note that only twin systems with $d_{m f p}^{s, t}<D$ are affected by this approach. When there are no twin lamellae present or the slip system is parallel to the twin plane $d_{m f p}^{s, t}=D$. 


\subsection{VPSC simulation inputs}

Since the VPSC model only simulates plastic deformation and does not account for elasticity, it is not possible to fit the initial elastic loading regime or the elastic unloading upon load reversal. The hardening parameters were adjusted until optimal correspondence with the plastic regime of the experimental stressstrain curve was achieved. The parameters utilised in the present simulation are listed in Tables 1 and 2.

The texture input for the simulations comprised 5000 discrete orientations calculated from the initial orientation distribution function obtained via X-ray diffraction. The deformation systems introduced into the VPSC model comprised the $\{111\}\langle 110\rangle$ slip systems and the $\{111\}\langle 112\rangle$ twinning systems. While the VPSC model allows for various grain-matrix interaction (homogenisation) schemes, the so-called $n^{e f f}=10$ intermediate interaction scheme (intermediate between the Taylor and Sachs bounds) was used for the present simulations.

\section{Results and discussion}

\subsection{Experimental observations}

Fig. 3 shows the experimental true stress vs. strain curve during forward tension loading (T) up to a true strain of 0.128 followed by reverse compression loading (TC) back to a true strain of 0.031 . Here, TC corresponds to a total accumulated true strain of 0.225 .

Fig. 4a shows the initial fully recrystallised microstructure, which is characterised by equiaxed grains of average grain size $5.8 \pm 4.5 \mu \mathrm{m}$ (without considering twin boundaries) and a high fraction of annealing twin boundaries ( $\sim 50 \%$ of the total grain boundary area fraction). Fig. $4 \mathrm{~b}$ shows the same area after forward tension loading (T) to 0.128 true strain. As indicated by the yellow ovals in Fig. $4 \mathrm{~b}$, deformation twins were found in the form of single lines or parallel packets and they evolve preferentially in grains with orientations close to $\langle 111\rangle$ and $\langle 110\rangle$ parallel to the tensile axis (see inset inverse pole figure in Fig. $4 \mathrm{~b}$ ). These deformation twins originate mostly from the grain boundaries or from annealing twin boundaries, while in a few instances they were observed to start and finish within a grain. Furthermore, some in-grain striations were also observed (refer to the white arrows in Fig. 4b). As mentioned before, previous studies have pointed out that the thickness of individual deformation twins is of the order of tens of nanometres such that they cannot be crystallographically detected via EBSD due to spatial resolution limitations [45]. When deformation-twinning activity increases such that the twins stack into relatively thick bundles, they can eventually be detected by EBSD. Alternatively, other studies have raised the possibility that some of these in-gran striations observed in band contrast maps could be stacking faults [46].

Subsequent reverse compression loading (TC) back to a true strain of 0.031 led to the removal of all deformation twins (as clearly seen by comparing the yellow ovals in Figs. 4b and 4c) along with the 
formation of in-grain striations (refer to the white arrows in Fig. 4c). The former observation provides unambiguous firsthand evidence of de-twinning during load reversal in TWIP steel.

Fig. 5 comprises representative inverse pole figure maps of grains 1, 2 and 3 marked in Fig. 4b after forward tension (Figs. 5a, 5d and 5g) and reverse compression loading (Figs. 5b, 5e and 5h). As described in the caption of Fig. 5, the orientations of grains 1, 2 and 3 are near the $\langle 111\rangle$, between $\langle 100\rangle$ and $\langle 111\rangle$ and near $\langle 110\rangle$ parallel to the tensile axis, respectively. The misorientation profiles along the white dashed lines show clear evidence of twinning and de-twinning after forward tension and reverse compression loading in all three grains (Figs. 5c, 5f and 5i). A further observation in grain 1 is that the annealing twin highlighted in Fig. 5a does not undergo de-twinning; likely due to the absence of a twinning-induced backstress acting on it.

The observation of de-twinning of deformation twins upon load reversal has been well documented in hcp materials $[8,9,47]$, where the slip and twinning systems are highly restricted due to the limited number of slip planes. This restriction in slip planes leads to the twinning system activated in the forward direction to be most likely to de-twin on reverse loading, due to the reverse glide of partials bounding the twin rather than activating new slip or twinning systems.

With respect to fcc materials, Szczerba et al. [20] observed de-twinning of deformation twins in $\mathrm{Cu}$ 8.5Al single crystal subjected to compression after generating deformation twins during pre-loading in tension. Using transmission electron microscopy, Szczerba et al. [20] showed that the partial dislocations bounding a deformation twin glide back and forth during loading and unloading. Upon reaching a local critical stress, the twin partials were able to glide in the reverse direction; resulting in a reconfiguration of the twinned region to match the parent grain and thereby, effectively removing the deformation twin. This mechanism was described as the reverse of twin formation.

De-twinning of deformation twins was possible within the $\mathrm{Cu}-8.5 \mathrm{Al}$ fcc single crystal [20] due to a restriction imposed by existing deformation twins on the number of operable slip planes. Therein, it was shown that the deformation twins restricted the transfer of shear perpendicular to the twin-matrix interface and limited the shear transfer in the parallel direction. The critical shear stress for dislocation propagation across the deformation twin was more than twice that required to activate slip parallel to the twin/matrix interface. This restricted glide is analogous to the restricted number of slip systems in hcp materials. Consequently, during reverse loading, only one twin system was activated. This restriction resulted in partial dislocations bounding the deformation twin to reverse direction, instead of activating another twin system [20], and led to de-twinning.

With respect to coarse grained polycrystalline fcc materials, a phase field modelling study [48] simulated the evolution of deformation twinning during loading and unloading for micron sized grains. The model showed that partials bounding a deformation twin could glide in the reverse direction upon 
unloading, allowing for the reorientation of deformation twins to match the parent grain. Similar to the above single crystal experimental study [20], the phase field simulations verified the possibility of the reverse glide of the partial dislocations bounding the deformation twin in polycrystalline structures.

\subsection{VPSC model predictions}

The simulated stress-strain curve is compared with its experimental counterpart in Fig. 3. It is noted that since the VPSC model does not account for elasticity, the elastic part was removed from the experimental stress-strain data in order to compare directly with the simulated curve. In general, the stressstrain curve is slightly over-predicted with increasing strain during forward tensile loading. The absence of elasticity from the model expectedly leads to the observed mismatch between experiment and simulation during unloading. Thereafter, the stress-strain curve is slightly under-predicted during the initial stages of reverse compression loading such that good agreement between experiment and simulation is obtained with greater reverse loading.

The relative slip, twinning and de-twinning activities as a function of the accumulated true strain are shown in Fig. 6a. During forward tensile loading, the VPSC model returns higher slip activity compared to twinning such that a monotonic decline in twinning is noted with increasing tensile strain. During the initial stages of reverse compression loading, deformation is accommodated solely via slip due to the strong backstress accumulated during forward loading facilitating slip during reverse loading. Here, since the previously activated slip systems experience a back-stress, they are easier to activate upon load reversal.

The subsequent decline in relative slip activity is associated with the concurrent increase in detwinning. In this case, the back-stress associated with previously activated twin systems leads to their opposite systems becoming softer, which in turn, facilitates de-twinning rather than activating new twinning systems. With further reverse compression loading, the observed gradual decrease in de-twinning activity is due to the elimination of twins that formed during forward tension loading.

It is also noted in Fig. 6a that twinning activity during reverse compression loading is lower than during forward tensile loading. This observation is in agreement with Schmid factor considerations wherein not all twinning systems activated during tension are activated during compression [49] as they are not favourably oriented. Furthermore, although VPSC predicts twinning activity during reverse loading, twinning was not observed in Fig. 4c. As mentioned previously, this disparity may be ascribed to the thickness of individual deformation twins being of the order of tens of nanometres such that they were not yet stacked into relatively thicker bundles detectable by EBSD.

As shown in Fig. $6 \mathrm{~b}$, less than $\sim 2 \%$ twin volume fraction is predicted at the end of forward tensile loading. This is in agreement with the limited twinning volume fraction typically reported for low SFE 
materials in general and TWIP steel in particular [45, 50-52]. The above limited twin volume fraction is also close to our visco-plastic self-consistent model prediction of $\sim 4.5 \%$ (at 0.128 true strain) following the simulation of the monotonic tensile loading of the same TWIP steel using the predominant twin reorientation scheme [45]. In this regard, the predominant twin reorientation scheme used in Ref. [45] tends to overestimate the twin volume fraction as it reorients the whole grain, when in reality, only the twinned part should be considered. As per the explanation for Fig. 6a, reverse compression loading results in a gradual decrease in the twin volume fraction due to de-twinning followed by a subsequent increase towards the end of reverse compression loading.

Fig. 6c shows the individual contributions of twinning (dashed green line) and de-twinning (dotted red line) to the change in twin volume fraction with the effective (solid black line) being the difference between the two mechanisms. During forward tensile loading, the effective and twinning lines obviously match each other. Alternatively, during reverse compression loading, when de-twinning is dominant, the effective and de-twinning lines closely follow each other. With the gradual elimination of the twins created during forward loading, a concomitant decline in de-twinning activity, the effective line gradually approaches the twinning line as twinning is activated again towards the end of reverse compression loading.

\section{Conclusions}

EBSD mapping of the same selected area provided the first direct experimental evidence of detwinning during interrupted reverse (tension-compression) loading of TWIP steel in particular, and low SFE coarse grained polycrystalline fcc materials in general. This de-twinning process upon load reversal can be ascribed to the possibility of the reverse glide of the partial dislocations bounding the deformation twin, which in turn leads to the reorientation of the twin to match the parent grain.

The reverse loading behaviour was successfully simulated by the RGBV-VPSC model supporting our formulation of the twinning back-stress and the twin barrier effect proposed to track the processes of twinning and de-twinning (twin lamellae generation/annihilation) during load reversal.

\section{Acknowledgments}

This work was funded by the Australian Research Council - Discovery Project (DP130101882). The authors thank Prof. D.B. Santos (UFMG, Brazil) for providing the source material and Mr. Heiko Tysar (BlueScope Steel Limited) for his help with mechanical testing. CNT was fully supported by the U.S. DOE project FWP 06SCPE401 under U.S. DOE contract W-7405-ENG-36. The JEOL JSM-7001F FEG-SEM was funded by the Australian Research Council Linkage Infrastructure, Equipment and Facilities grant LE0882613. 


\section{References}

[1] O. Grassel, L. Kruger, G. Frommeyer, L.W. Meyer, High strength Fe-Mn-(Al, Si) TRIP/TWIP steels development - properties - application, Int. J. Plasticity 16 (2000) 1391-1409.

[2] T.S. Byun, On the stress dependence of partial dislocation separation and deformation microstructure in austenitic stainless steels, Acta Mater. 51 (2003) 3063 - 3071.

[3] S.M. Copley, B.H. Kear, The dependence of the width of a dissociated dislocation on dislocation velocity, Acta Metall. 16 (1968) 227-231.

[4] D. Goodchild, W. Roberts, D. Wilson, Plastic deformation and phase transformation in textured austenitic stainless steel, Acta Mater. 18 (1970) 1137.

[5] J. Kestenbach, The effect of applied stress on partial dislocation separation and dislocation substructure in austenitic strainless steels, Philos. Mag. 36 (1977) 1509 - 1515.

[6] L. Wu, S.R. Agnew, D.W. Brown, G.M. Stoica, B. Clausen, A. Jain, D.E. Fielden, P.K. Liaw, Internal stress relaxation and load redistribution during the twinning-detwinning-dominated cyclic deformation of a wrought magnesium alloy, ZK60A, Acta Mater. 56 (2008) 3699-3707.

[7] G. Proust, G.C. Kaschner, I.J. Beyerlein, B. Clausen, D.W. Brown, R.J. McCabe, C.N. Tomé, Detwinning of High-Purity Zirconium: In-Situ Neutron Diffraction Experiments, Experimental Mechanics 50 (2010) 125-133.

[8] H. Wang, P.D. Wu, C.N. Tomé, J. Wang, A constitutive model of twinning and detwinning for hexagonal close packed polycrystals, Mater. Sci. Eng. A 555 (2012) 93-98.

[9] D. Sarker, D.L. Chen, Detwinning and strain hardening of an extruded magnesium alloy during compression, Scripta Mater. 67 (2012) 165-168.

[10] H. Wang, P.D. Wu, J. Wang, C.N. Tomé, A crystal plasticity model for hexagonal close packed (HCP) crystals including twinning and de-twinning mechanisms, Int. J. Plasticity 49 (2013) 36-52. [11] L. Balogh, S.R. Niezgoda, A.K. Kanjarla, D.W. Brown, B. Clausen, W. Liu, C.N. Tomé, Spatially resolved in situ strain measurements from an interior twinned grain in bulk polycrystalline AZ31 alloy, Acta Mater. 61 (2013) 3612-3620.

[12] M. Arul Kumar, A.K. Kanjarla, S.R. Niezgoda, R.A. Lebensohn, C.N. Tomé, Numerical study of the stress state of a deformation twin in magnesium, Acta Mater. 84 (2015) 349-358.

[13] M. Arul Kumar, I.J. Beyerlein, R.J. McCabe, C.N. Tomé, Grain neighbour effects on twin transmission in hexagonal close-packed materials, Nat. Commun. 7 (2016) 13826.

[14] M. Arul Kumar, I.J. Beyerlein, C.N. Tomé, Effect of local stress fields on twin characteristics in HCP metals, Acta Mater. 116 (2016) 143-154.

[15] H. Abdolvand, M. Majkut, J. Oddershede, S. Schmidt, U. Lienert, B.J. Diak, P.J. Withers, M.R. Daymond, On the deformation twinning of Mg AZ31B: A three-dimensional synchrotron X-ray diffraction experiment and crystal plasticity finite element model, Int. J. Plasticity 70 (2015) 77-97. [16] T.R. Bieler, L. Wang, A.J. Beaudoin, P. Kenesei, U. Lienert, In Situ Characterization of Twin Nucleation in Pure Ti Using 3D-XRD, Metall. Mater. Trans. A 45 (2014) 109-122.

[17] S.B. Chakrabortty, T.K. Mukhopadhyay, E.A. Starke Jr, The cyclic stress-strain response of titanium-vanadium alloys, Acta Metall. 26 (1978) 909-920.

[18] S. Ni, Y.B. Wang, X.Z. Liao, H.Q. Li, R.B. Figueiredo, S.P. Ringer, T.G. Langdon, Y.T. Zhu, Effect of grain size on the competition between twinning and detwinning in nanocrystalline metals, Phys. Rev. B 84 (2011) 235401.

[19] L. Li, T. Ungár, Y.D. Wang, J.R. Morris, G. Tichy, J. Lendvai, Y.L. Yang, Y. Ren, H. Choo, P.K. Liaw, Microstructure evolution during cold rolling in a nanocrystalline $\mathrm{Ni}-\mathrm{Fe}$ alloy determined by synchrotron X-ray diffraction, Acta Mater. 57 (2009) 4988 - 5000.

[20] M.S. Szczerba, S. Kopacz, M.J. Szczerba, On the reverse Mode of FCC deformation twinning, Acta Mater. 60 (2012) 6413 - 6420. 
[21] V. Doquet, Twinning and multiaxial cyclic plasticity of a low stacking-fault-energy f.c.c. alloy, Acta Metall. Mater. 41 (1993) 2451-2459.

[22] C.C. Aydıner, J.V. Bernier, B. Clausen, U. Lienert, C.N. Tomé, D.W. Brown, Evolution of stress in individual grains and twins in a magnesium alloy aggregate, Phys. Rev. B 80 (2009) 024113.

[23] L. Wang, J. Lind, H. Phukan, P. Kenesei, J.S. Park, R.M. Suter, A.J. Beaudoin, T.R. Bieler, Mechanical twinning and detwinning in pure Ti during loading and unloading - An in situ highenergy X-ray diffraction microscopy study, Scripta Mater. 92 (2014) 35-38.

[24] G. Proust, C.N. Tomé, A. Jain, S.R. Agnew, Modeling the effect of twinning and detwinning during strain-path changes of magnesium alloy AZ31, Int. J. Plasticity 25 (2009) 861-880.

[25] H. Qiao, S.R. Agnew, P.D. Wu, Modeling twinning and detwinning behavior of Mg alloy ZK60A during monotonic and cyclic loading, Int. J. Plasticity 65 (2015) 61-84.

[26] C.F. Gu, L.S. Toth, M. Hoffman, Twinning effects in a polycrystalline magnesium alloy under cyclic deformation, Acta Mater. 62 (2014) 212-224.

[27] A.A. Saleh, B. Clausen, D.W. Brown, E.V. Pereloma, C.H.J. Davies, C.N. Tomé, A.A. Gazder, On the feasibility of partial slip reversal and de-twinning during the cyclic loading of TWIP steel, Mater. Lett. 182 (2016) 294-297.

[28] G. Palumbo, K. Aust, Structure-dependence of intergranular corrosion in high purity nickel, Acta Metall. Mater. 38 (1990) 2343 - 2352.

[29] E.F. Rauch, J.J. Gracio, F. Barlat, G. Vincze, Modelling the plastic behaviour of metals under complex loading conditions, Modell. Simul. Mater. Sci. Eng. 19 (2011) 035009.

[30] K. Kitayama, C.N. Tomé, E.F. Rauch, J.J. Gracio, F. Barlat, A crystallographic dislocation model for describing hardening of polycrystals during strain path changes. Application to low carbon steels, Int. J. Plasticity 46 (2013) 54-69.

[31] W. Wen, M. Borodachenkova, C.N. Tomé, G. Vincze, E.F. Rauch, F. Barlat, J.J. Grácio, Mechanical behavior of Mg subjected to strain path changes: Experiments and modeling, Int. J. Plasticity 73 (2015) 171-183.

[32] W. Wen, M. Borodachenkova, C.N. Tomé, G. Vincze, E.F. Rauch, F. Barlat, J.J. Grácio, Mechanical behavior of low carbon steel subjected to strain path changes: Experiments and modeling, Acta Mater. 111 (2016) 305-314.

[33] I.J. Beyerlein, C.N. Tomé, A dislocation-based constitutive law for pure Zr including temperature effects, Int. J. Plasticity 24 (2008) 867-895.

[34] O. Bouaziz, S. Allain, C. Scott, Effect of grain and twin boundaries on the hardening mechanisms of twinning-induced plasticity steels, Scripta Mater. 58 (2008) 484-487.

[35] B. Peeters, S.R. Kalidindi, P. Van Houtte, E. Aernoudt, A crystal plasticity based workhardening/softening model for b.c.c. metals under changing strain paths, Acta Mater. 48 (2000) 2123-2133.

[36] B. Peeters, M. Seefeldt, C. Teodosiu, S.R. Kalidindi, P. Van Houtte, E. Aernoudt, Workhardening/softening behaviour of b.c.c. polycrystals during changing strain paths: I. An integrated model based on substructure and texture evolution, and its prediction of the stress-strain behaviour of an IF steel during two-stage strain paths, Acta Mater. 49 (2001) 1607-1619.

[37] L. Kubin, B. Devincre, T. Hoc, Modeling dislocation storage rates and mean free paths in facecentered cubic crystals, Acta Mater. 56 (2008) 6040-6049.

[38] N. Bertin, C.N. Tomé, I.J. Beyerlein, M.R. Barnett, L. Capolungo, On the strength of dislocation interactions and their effect on latent hardening in pure Magnesium, Int. J. Plasticity 62 (2014) 7292.

[39] U.F. Kocks, H. Mecking, Physics and phenomenology of strain hardening: the FCC case, Prog. Mater Sci. 48 (2003) 171-273. 
[40] R.A. Lebensohn, C.N. Tomé, A self-consistent anisotropic approach for the simulation of plastic deformation and texture development of polycrystals: Application to zirconium alloys, Acta Metall. Mater. 41 (1993) 2611-2624.

[41] R.A. Lebensohn, C.N. Tomé, P.P. CastaÑeda, Self-consistent modelling of the mechanical behaviour of viscoplastic polycrystals incorporating intragranular field fluctuations, Philos. Mag. 87 (2007) 4287-4322.

[42] M. Arul Kumar, I.J. Beyerlein, R.A. Lebensohn, C.N. Tomé, Modeling the effect of neighboring grains on twin growth in HCP polycrystals, Modell. Simul. Mater. Sci. Eng. 25 (2017) 064007.

[43] B. Clausen, C.N. Tomé, D.W. Brown, S.R. Agnew, Reorientation and stress relaxation due to twinning: Modeling and experimental characterization for Mg, Acta Mater. 56 (2008) 2456-2468. [44] S. Allain, J.P. Chateau, D. Dahmoun, O. Bouaziz, Modeling of mechanical twinning in a high manganese content austenitic steel, Mater. Sci. Eng. A 387-389 (2004) 272-276.

[45] A.A. Saleh, E.V. Pereloma, A.A. Gazder, Microstructure and texture evolution in a twinninginduced-plasticity steel during uniaxial tension, Acta Mater. 61 (2013) 2671-2691.

[46] A.A. Gazder, A.A. Saleh, A.G. Kostryzhev, E.V. Pereloma, Application of Transmission Kikuchi Diffraction to a Multi-phase TRIP-TWIP Steel, Materials Today: Proceedings 2 (2015) S647-S650. [47] J. Wang, I. Beyerlein, N. Mara, A. Misra, C. Tome, Deformation twinning mechanisms in FCC and HCP metals, Advances in Heterogeneous Material Mechanaics Shanghai, 2011.

[48] S. Hu, C.H. Jr, L. Chen, Simulations of stress-induced twinning and de-twinning: A phase field model, Acta Mater. (2010) 6554 - 6564.

[49] W.F. Hosford, The Mechanics of Crystals and Textured Polycrystals, Oxford University Press1993.

[50] T. Leffers, R.K. Ray, The brass-type texture and its deviation from the copper-type texture, Prog. Mater Sci. 54 (2009) 351-396.

[51] A.A. Saleh, E.V. Pereloma, B. Clausen, D.W. Brown, C.N. Tomé, A.A. Gazder, On the evolution and modelling of lattice strains during the cyclic loading of TWIP steel, Acta Mater. 61 (2013) 52475262.

[52] A.A. Saleh, E.V. Pereloma, B. Clausen, D.W. Brown, C.N. Tomé, A.A. Gazder, Self-consistent modelling of lattice strains during the in-situ tensile loading of twinning induced plasticity steel, Mater. Sci. Eng. A 589 (2014) 66-75. 


\section{Figures}

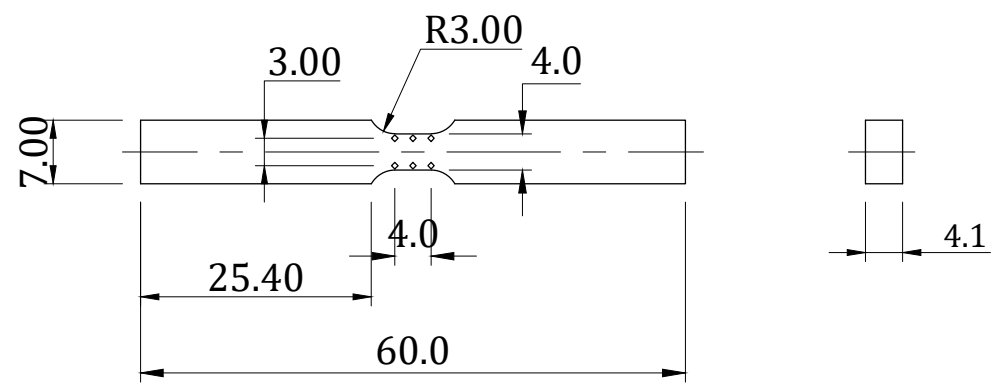

Figure 1. Dimensions (in $\mathrm{mm}$ ) of the dog-bone sample used for forward tension - reverse compression loading. The gauge length is parallel to the rolling direction.

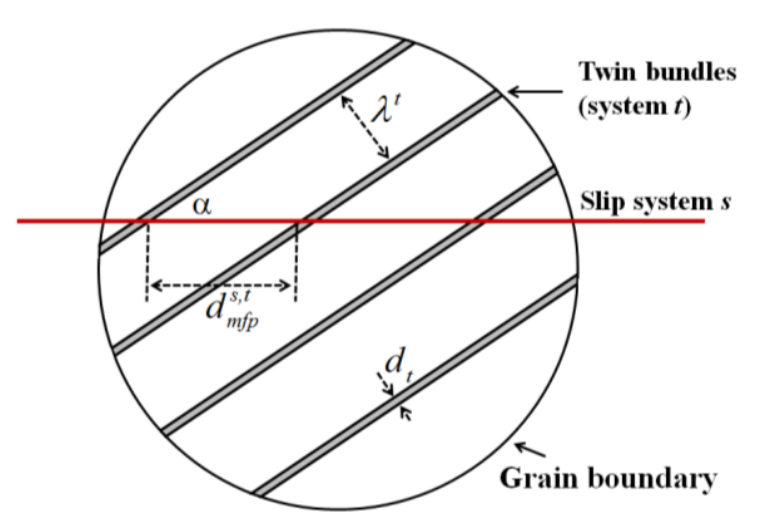

(a)

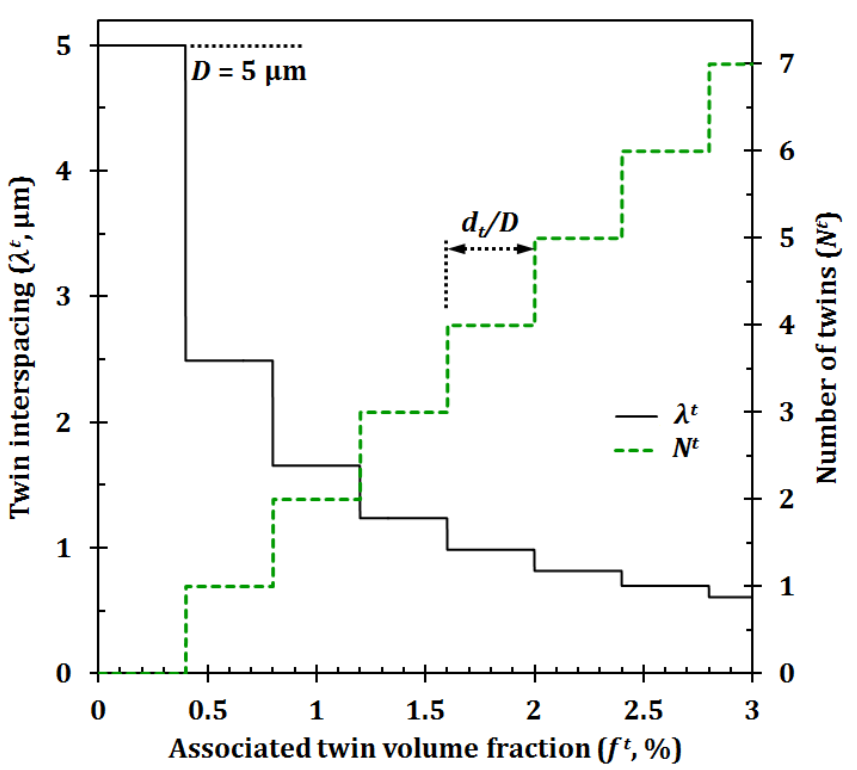

(b)

Figure 2. (a) Schematic of the twin-matrix microstructure showing the characteristic lengths used in the VPSC model. (b) Evolution of the number of twin lamellae and their interspacing as a function of the associated twin volume fraction during forward loading. 


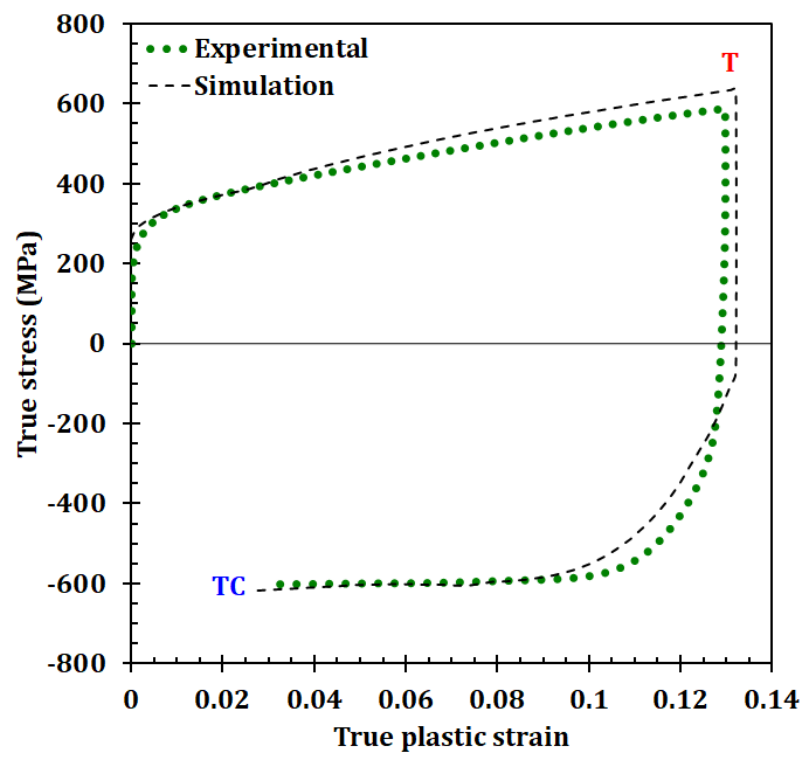

Figure 3. The experimental and VPSC simulated true stress vs. true plastic strain during forward tension (T) - reverse compression (TC) loading. 
(a)

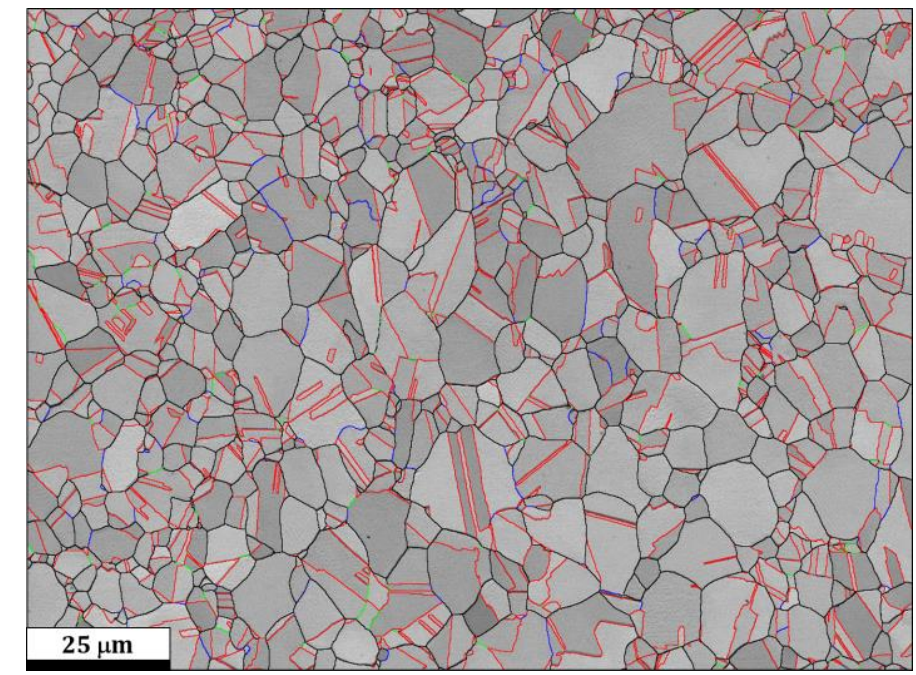

(b)

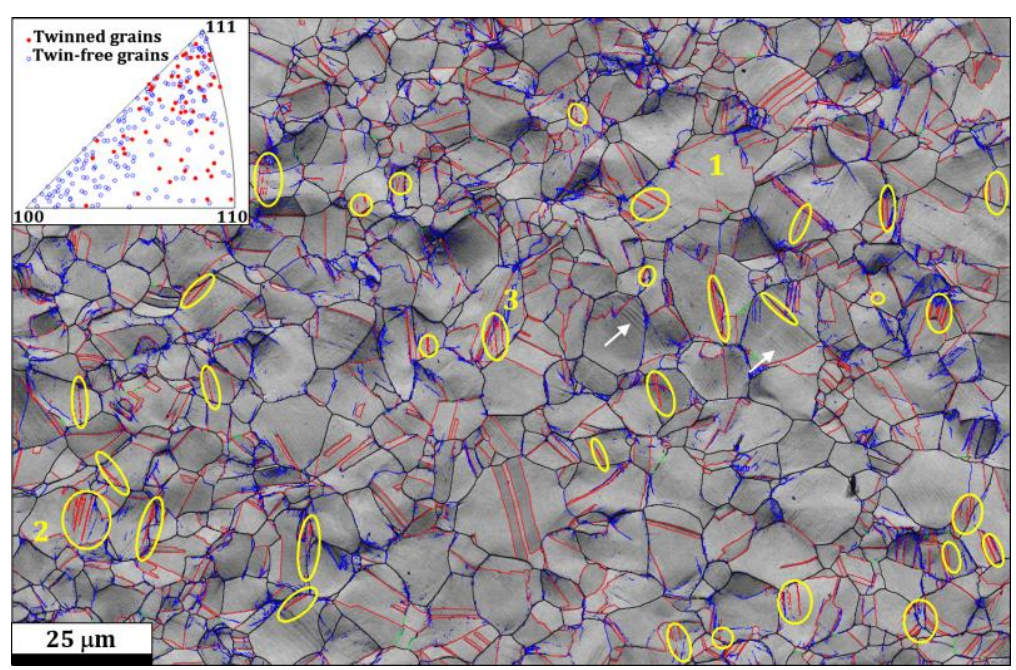

(c)

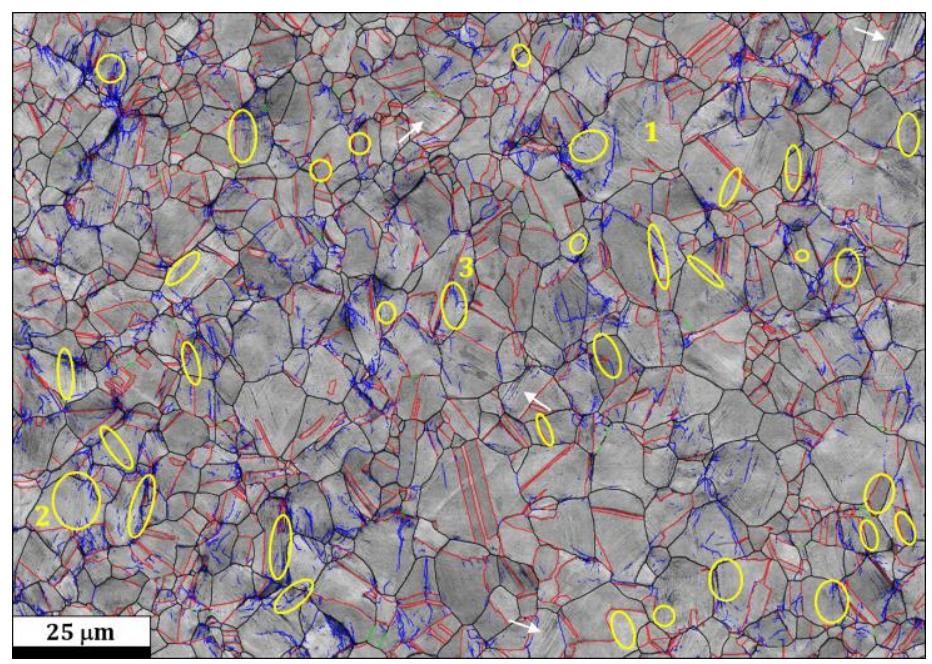

Figure 4. Band contrast maps at true strains of (a) 0 , initial, (b) 0.128, after forward tension loading (T) and (c) 0.031, after reverse compression loading (TC). LAGBs = blue, HAGB = black, $\Sigma 3$ $\left(60^{\circ}\langle 111\rangle\right)=\operatorname{red}$ and $\Sigma 9\left(38.9^{\circ}\langle 110\rangle\right)=$ green. The yellow ellipses indicate regions where twinning and de-twinning were observed. The loading direction is horizontal. 


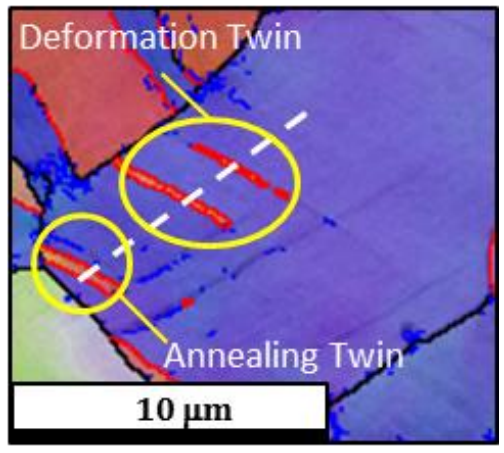

(a)

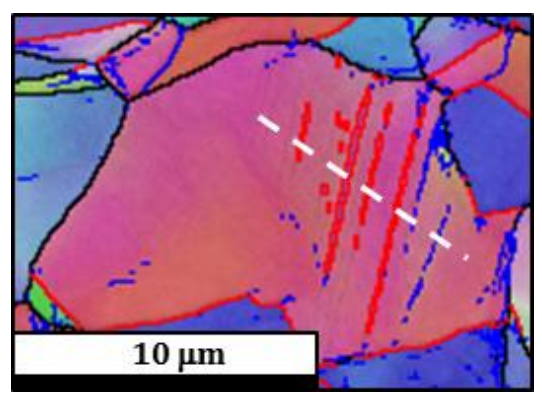

(d)

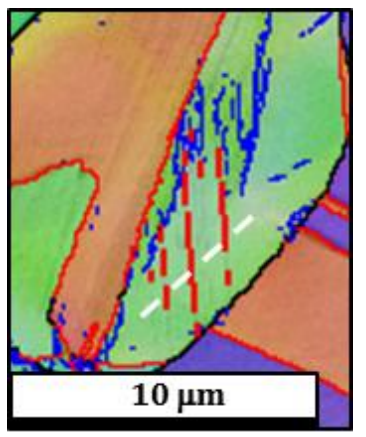

(g)

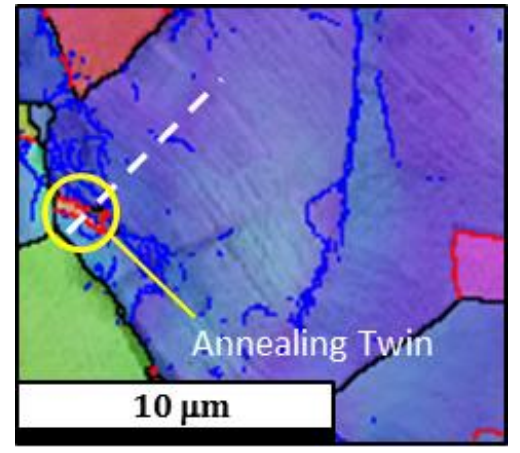

(b)

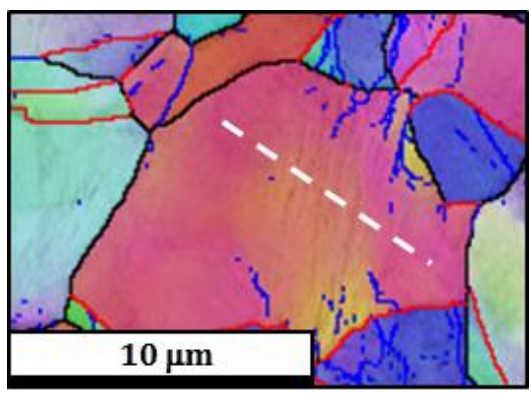

(e)

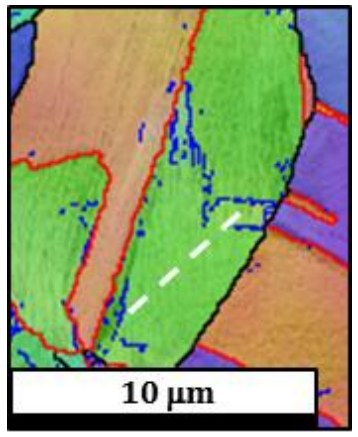

(h)

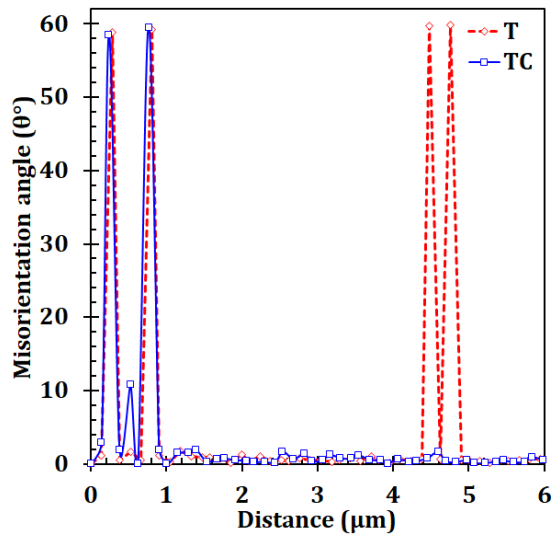

(c)

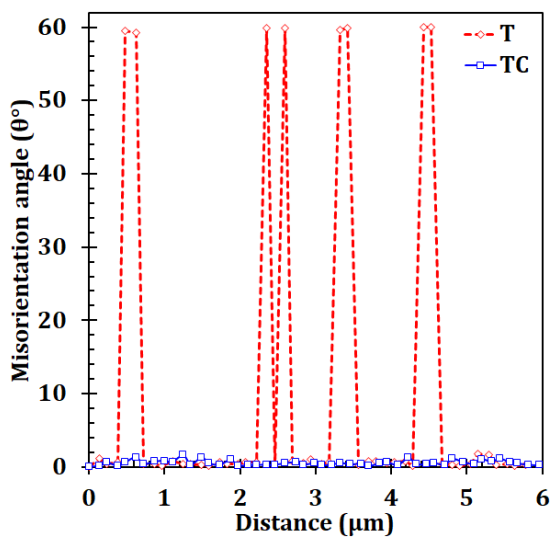

(f)

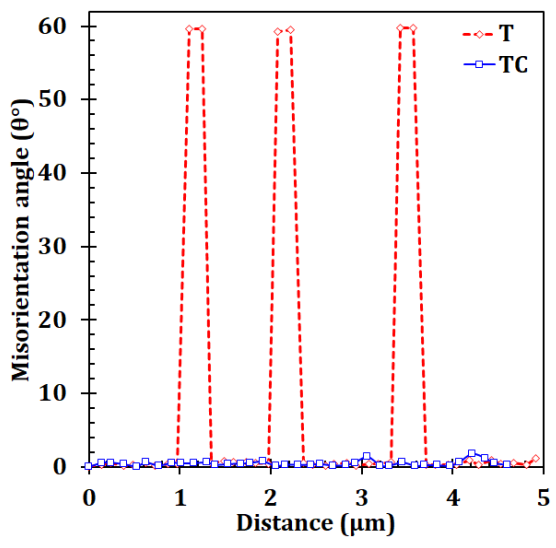

(i)

Figure 5. Representative inverse pole figure maps of grains 1, 2 and 3 marked in Fig. $4 \mathrm{~b}$ after $(\mathrm{a}, \mathrm{d}, \mathrm{g})$ forward tension and $(\mathrm{b}, \mathrm{e}, \mathrm{h})$ reverse compression loading. (c, f, i) Misorientation profiles along the white dashed lines evidence twinning and detwinning after forward tension (T) and reverse compression (TC) loading. Grain $1=$ $(134)\langle 1 \overline{2} 1\rangle$ which is near $\langle 111\rangle$, grain $2=(011)\langle 51 \overline{1}\rangle$ which is between $\langle 100\rangle$ and $\langle 111\rangle$ and grain $3=(127)\langle 1 \overline{2} 1\rangle$ which is near $\langle 110\rangle$. LAGBs $=$ blue, HAGB = black and

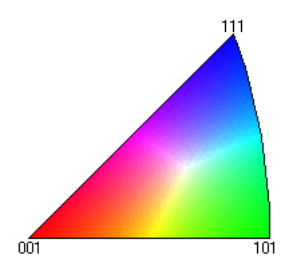
$\Sigma 3\left(60^{\circ}\langle 111\rangle\right)=$ red. The loading direction is horizontal. 


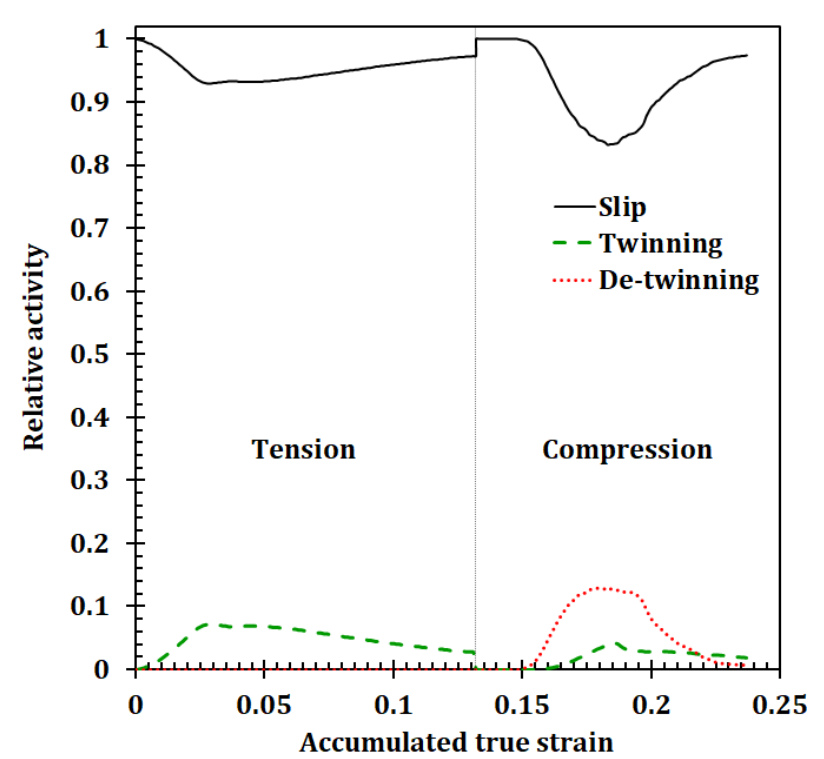

(a)

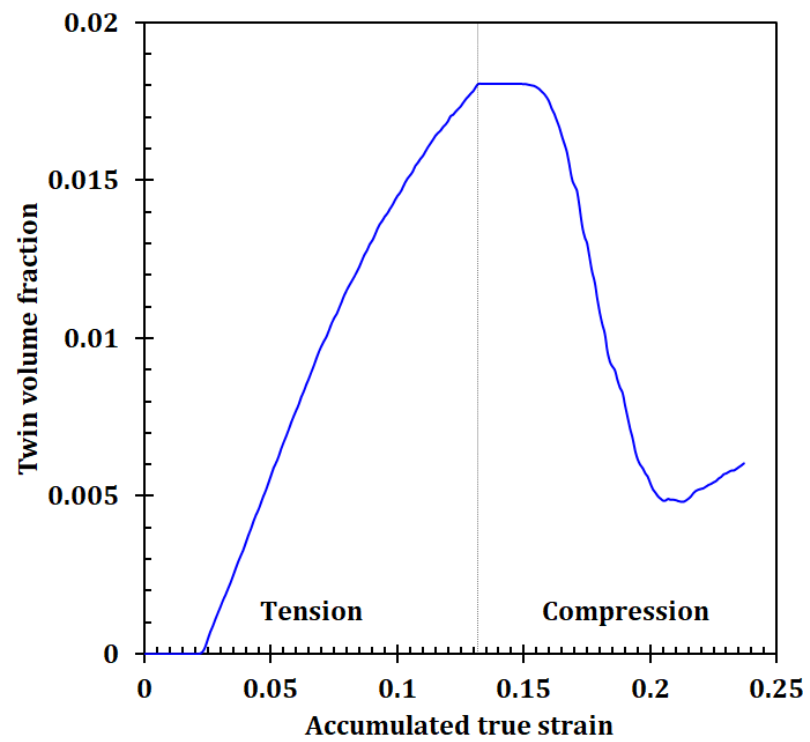

(b)

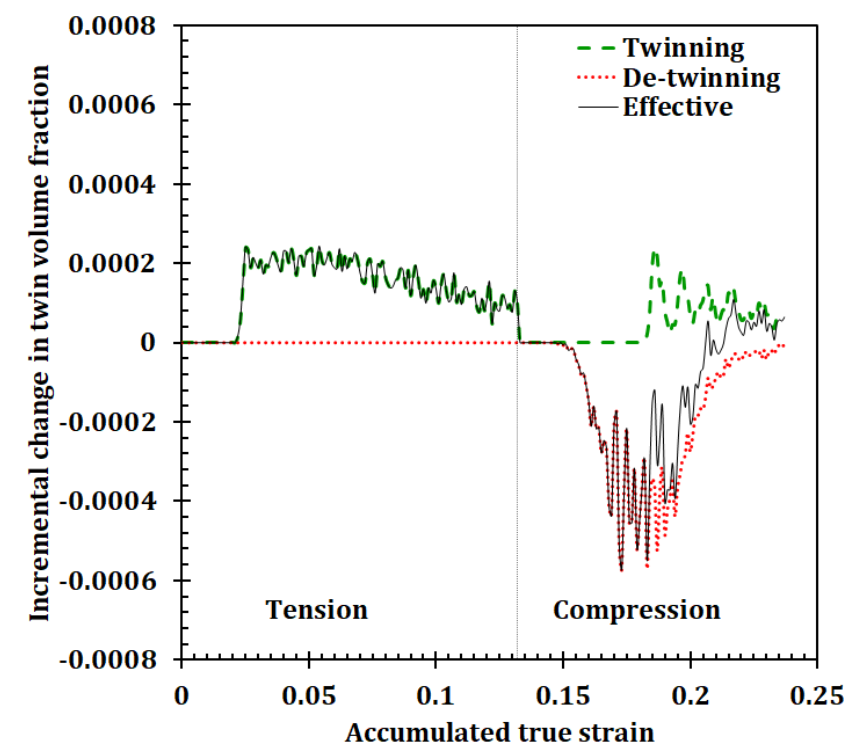

(c)

Figure 6. VPSC model predictions of the (a) relative slip, twinning and de-twinning activities, (b) volume fraction of twins and (c) change in the twin volume fraction due to twinning and de-twinning activities. 


\section{Tables}

Table 1. The parameters used in the RGBV-VPSC model for the present TWIP steel.

\begin{tabular}{ll}
\hline Parameter & Value \\
\hline$\tau_{0}$ (Initial CRSS) & $50 \mathrm{MPa}$ (slip) and $40 \mathrm{MPa}$ (twinning) \\
$b^{\alpha}$ (Burgers vector) & $2.5 \times 10^{-10} \mathrm{~m}$ (slip) and $1.5 \times 10^{-10} \mathrm{~m}$ (twinning) \\
$D$ (Grain size) & $5 \mu \mathrm{m}$ \\
$f$ (Recombination parameter) & 1.0 \\
$\mu$ (Shear modulus) & $76 \mathrm{GPa}$ \\
$K$ (Mobile to storage parameter) & 400 \\
$q$ (Back-stress power parameter) & 5 \\
$f_{B}^{S}$ (Back-stress parameter) & 0.9 \\
$m$ (Recombination rate parameter) & 0.3 \\
$\rho_{\text {min }}$ (Lower reversibility threshold) & $10^{12} \mathrm{~m}^{-2}$ \\
$\rho_{\text {max }}$ (Upper reversibility threshold) & $7 \times 10^{14} \mathrm{~m}^{-2}$ \\
$S$ (Characteristic twinning shear) & 0.707 \\
$\alpha^{s s^{\prime}}$ (Dislocation interaction matrix) & 0.25 \\
$C^{\beta \alpha}$ (Twin-slip interactions) & 150 \\
$\varphi$ (Twin back-stress scaling parameter) & 0.0186 \\
\hline
\end{tabular}

Table 2. $d_{m f p}^{s}$ related parameters.

\begin{tabular}{ll}
\hline Parameter & Value \\
\hline$H P^{S}$ & 0.158 \\
$H P^{s t}$ & 0.158 \\
$H P^{t}$ & $0.4 \mathrm{MPa} \mathrm{m}^{1 / 2}$ \\
$H P^{t t^{\prime}}$ & $0.6 \mathrm{MPa} \mathrm{m}^{1 / 2}$ \\
$d t$ & $20 \mathrm{~nm}$ \\
\hline
\end{tabular}

